\title{
Sharp geometrical properties of $a$-rarefied sets via fixed point index for the Schrödinger operator equations
}

\section{Zhiqiang $\mathrm{Li}^{1}$ and Beatriz Ychussie $\mathrm{2}^{*}$}

\section{"Correspondence:}

ychussie.b@gmail.com

${ }^{2}$ Mathematics Institute, Roskilde University, Roskilde, 4000, Denmark

Full list of author information is

available at the end of the article

\begin{abstract}
In this paper, we use the theory of fixed point index for the si ödinger operator equations to obtain a geometrical property of $a$-raref $t$ sets at. Kity on cones. Meanwhile, we give an example to show that the $r$ vers $f$ this property is not true.

Keywords: Schrödinger operator equations; an set; Pرsisson-Sch integral; Green-Sch potential
\end{abstract}

\section{Introduction and main theorem}

Let $\mathbf{R}$ and $\mathbf{R}_{+}$be the set of rea mbers and the set of all positive real numbers, respectively. We denote by $\mathbf{R}^{n}(, 2)$ ne $n$-dimensional Euclidean space. A point in $\mathbf{R}^{n}$ is denoted by $P=\left(X, x_{r}, X-\left(x_{1}, x_{2}, \ldots, x_{n-1}\right)\right.$. The Euclidean distance between two points $P$ and $Q$ in $\mathbf{R}^{n}$ is der tea $|P-Q|$. Also $|P-O|$ with the origin $O$ of $\mathbf{R}^{n}$ is simply denoted by $|P|$. The bo a 'ry and ne closure of a set $S$ in $\mathbf{R}^{n}$ are denoted by $\partial S$ and $\bar{S}$, respectively. For $P \in \mathbf{R}^{n}$ a. $\quad r>0$, let $B(P, r)$ denote the open ball with center at $P$ and radius $r$ in $\mathbf{R}^{n}$.

We ir aduce $\lambda$ system of spherical coordinates $(r, \Theta), \Theta=\left(\theta_{1}, \theta_{2}, \ldots, \theta_{n-1}\right)$, in $\mathbf{R}^{n}$ which a relatea $w$ Cartesian coordinates $\left(x_{1}, x_{2}, \ldots, x_{n-1}, x_{n}\right)$ by $x_{n}=r \cos \theta_{1}$.

el an arbitrary domain in $\mathbf{R}^{n}$ and $\mathscr{A}_{a}$ denote the class of nonnegative radial potentials $a(P)$, i.e. $0 \leq a(P)=a(r), P=(r, \Theta) \in D$, such that $a \in L_{\mathrm{loc}}^{b}(D)$ with some $b>n / 2$ if 4 and with $b=2$ if $n=2$ or $n=3$.

If $a \in \mathscr{A}_{a}$, then the Schrödinger operator

$$
\operatorname{Sch}_{a}=-\Delta+a(P) I=0
$$

where $\Delta$ is the Laplace operator and $I$ is the identical operator, can be extended in the usual way from the space $C_{0}^{\infty}(D)$ to an essentially self-adjoint operator on $L^{2}(D)$ (see [1], Chapter 11). We will denote it by $\operatorname{Sch}_{a}$ as well. This last one has a Green-Sch function $G_{D}^{a}(P, Q)$. Here $G_{D}^{a}(P, Q)$ is positive on $D$ and its inner normal derivative $\partial G_{D}^{a}(P, Q) / \partial n_{Q} \geq$ 0 , where $\partial / \partial n_{Q}$ denotes the differentiation at $Q$ along the inward normal into $D$.

We call a function $u \neq \equiv-\infty$ that is upper semi-continuous in $D$ a subfunction with respect to the Schrödinger operator $S_{c h}$ if its values belong to the interval $[-\infty, \infty)$ and at

(c) $2015 \mathrm{Li}$ and Ychussie. This article is distributed under the terms of the Creative Commons Attribution 4.0 International License (http://creativecommons.org/licenses/by/4.0/), which permits unrestricted use, distribution, and reproduction in any medium, provided you give appropriate credit to the original author(s) and the source, provide a link to the Creative Commons license, and indicate if changes were made. 
each point $P \in D$ with $0<r<r(P)$ the generalized mean-value inequality (see [2])

$$
u(P) \leq \int_{\partial B(P, r)} u(Q) \frac{\partial G_{B(P, r)}^{a}(P, Q)}{\partial n_{Q}} d \sigma(Q)
$$

is satisfied, where $G_{B(P, r)}^{a}(P, Q)$ is the Green-Sch function of $S_{c h}$ in $B(P, r)$ and $d \sigma(Q)$ is a surface measure on the sphere $\partial B(P, r)$.

If $-u$ is a subfunction, then we call $u$ a superfunction. If a function $u$ is both subfunction and superfunction, it is, clearly, continuous and is called a generalized harmonic functic (with respect to the Schrödinger operator $S c h_{a}$ ).

The unit sphere and the upper half unit sphere in $\mathbf{R}^{n}$ are denoted by $\mathbf{S}^{n-1}=\mathrm{d} \mathbf{S}_{+}^{n-1}$, respectively. For simplicity, a point $(1, \Theta)$ on $\mathbf{S}^{n-1}$ and the set $\{\Theta ;(1, \Theta) \in\langle\}\}$ $\Omega, \Omega \subset \mathbf{S}^{n-1}$, are often identified with $\Theta$ and $\Omega$, respectively. For two scts $\varepsilon \quad \mathbf{R}_{+}$and $\Omega \subset \mathbf{S}^{n-1}$, the set $\left\{(r, \Theta) \in \mathbf{R}^{n} ; r \in \Xi,(1, \Theta) \in \Omega\right\}$ in $\mathbf{R}^{n}$ is simply de 10 by $\Xi, \Omega$. By $C_{n}(\Omega)$, we denote the set $\mathbf{R}_{+} \times \Omega$ in $\mathbf{R}^{n}$ with the domain $\Omega$ on $\mathbf{S}^{n-1}$. We ca. a cone. We denote the set $I \times \Omega$ with an interval on $\mathbf{R}$ by $C_{n}(\Omega ; I)$.

We shall say that a set $H \subset C_{n}(\Omega)$ has a covering $\left\{r_{j}, R_{j}\right\}$ if then Yists a sequence of balls $\left\{B_{j}\right\}$ with centers in $C_{n}(\Omega)$ such that $H \subset \bigcup_{j=0}^{\infty} B_{j}$, where is the ra aus of $B_{j}$ and $R_{j}$ is the distance from the origin to the center of $B_{j}$. For positive fur $\alpha_{\text {ns }} h_{1}$ and $h_{2}$, we say that $h_{1} \lesssim h_{2}$ if $h_{1} \leq M h_{2}$ for some constant $M>0$. If $h_{1} \lesssim h_{2}$ an $h_{2} \lesssim h_{1}$, we say that $h_{1} \approx h_{2}$.

From now on, we always assume $D=C(\Omega)$. the sake of brevity, we shall write $G_{\Omega}^{a}(P, Q)$ instead of $G_{C_{n}(\Omega)}^{a}(P, Q)$. Throughou $\quad$ is pa er, let $c$ denote various positive constants, because we do not need to sp cify them. oreover, $\epsilon$ appearing in the expression in the following all sections will b a s. cient $y$ small positive number.

Let $\Omega$ be a domain on $\mathbf{S}^{n-1}, \quad$ smootr. oundary. Consider the Dirichlet problem

$$
\begin{aligned}
& \left(\Lambda_{n}+\lambda\right) \varphi=0 \text { or } \Omega, \\
& \varphi=0 \quad \text { on } \partial \Omega,
\end{aligned}
$$

where $\Lambda_{n}$ is the sp beric $\mu$ part of the Laplace operator $\Delta_{n}$ :

$$
\Delta_{n}=\frac{\partial^{2}}{\partial r}+\frac{\Lambda_{n}}{\partial r^{2}}
$$

We a te the least positive eigenvalue of this boundary value problem by $\lambda$ and the normalized positive eigenfunction corresponding to $\lambda$ by $\varphi(\Theta)$. In order to ensure the existence of $\lambda$ and a smooth $\varphi(\Theta)$. We put a rather strong assumption on $\Omega$ : if $n \geq 3$, then $\Omega$ is a $C^{2, \alpha}$-domain $(0<\alpha<1)$ on $\mathbf{S}^{n-1}$ surrounded by a finite number of mutually disjoint closed hypersurfaces.

Solutions of an ordinary differential equation

$$
-Q^{\prime \prime}(r)-\frac{n-1}{r} Q^{\prime}(r)+\left(\frac{\lambda}{r^{2}}+a(r)\right) Q(r)=0, \quad 0<r<\infty .
$$

It is well known (see, for example, [3]) that if the potential $a \in \mathscr{A}_{a}$, then (1.1) has a fundamental system of positive solutions $\{V, W\}$ such that $V$ and $W$ are increasing and decreasing, respectively (see [4-7]). 
We will also consider the class $\mathscr{B}_{a}$, consisting of the potentials $a \in \mathscr{A}_{a}$ such that there exists the finite limit $\lim _{r \rightarrow \infty} r^{2} a(r)=k \in[0, \infty)$, and moreover, $r^{-1}\left|r^{2} a(r)-k\right| \in L(1, \infty)$. If $a \in \mathscr{B}_{a}$, then the (sub)superfunctions are continuous (see [8]).

In the rest of paper, we assume that $a \in \mathscr{B}_{a}$ and we shall suppress this assumption for simplicity.

Denote

$$
\iota_{k}^{ \pm}=\frac{2-n \pm \sqrt{(n-2)^{2}+4(k+\lambda)}}{2},
$$

then the solutions to (1.1) have the asymptotics (see [9])

$$
V(r) \approx r_{k}^{\iota_{k}^{+}}, \quad W(r) \approx r^{l_{k}}, \quad \text { as } r \rightarrow \infty
$$

Let $v$ be any positive measure on cones such that the Green-Sch potent.

$$
G_{\Omega}^{a} v(P)=\int_{C_{n}(\Omega)} G_{\Omega}^{a}(P, Q) d v(Q) \not \equiv+\infty
$$

for any $P \in C_{n}(\Omega)$. Then the positive measure $v^{\prime}$ on $\mathbf{R}^{n}$ is ${ }^{2}$ efined by

$$
d \nu^{\prime}(Q)= \begin{cases}W(t) \varphi(\Phi) d \nu(Q), & Q=\left(t, 2=C_{n}(-(1,+\infty)),\right. \\ 0, & \left.Q \in \mathbb{N}^{n}-C_{n}:(1,+\infty)\right) .\end{cases}
$$

The Poisson-Sch integral $P I_{\Omega}^{a},(P) \not \equiv+\quad\left(P \in C_{n}(\Omega)\right)$ of $\mu$ on cones is defined as follows:

where

$$
\left.P I_{\Omega}^{a} \mu(P)=\frac{1}{c_{n}} \int_{S_{n}(} P_{\Omega}^{a}(P, Q) d,, Q\right)
$$

$$
P I^{a}(P,)=\frac{\partial \sum_{\Omega}^{a}(P, Q)}{\partial n_{Q}}, \quad c_{n}= \begin{cases}2 \pi, & n=2, \\ (n-2) s_{n}, & n \geq 3,\end{cases}
$$

$\mu$ positive measure on $\partial C_{n}(\Omega)$ and $\partial / \partial n_{Q}$ denotes the differentiation at $Q$ along the inwa ormal into cones. Then the positive measure $\mu^{\prime}$ on $\mathbf{R}^{n}$ is defined by

$$
d \mu^{\prime}(Q)= \begin{cases}t^{-1} W(t) \frac{\partial \varphi(\Phi)}{\partial n_{\Phi}} d \mu(Q), & Q=(t, \Phi) \in S_{n}(\Omega ;(1,+\infty)) \\ 0, & Q \in \mathbf{R}^{n}-S_{n}(\Omega ;(1,+\infty))\end{cases}
$$

Remark We remark that the total masses of $\mu^{\prime}$ and $v^{\prime}$ are finite (see [2], Lemma 5 and [6], Lemma 4).

Let $0 \leq \alpha \leq n$ and $\lambda$ be any positive measure on $\mathbf{R}^{n}$ having finite total mass. For each $P=(r, \Theta) \in \mathbf{R}^{n}-\{O\}$, the maximal function $M(P ; \lambda, \alpha)$ with respect to $S_{c h}$ is defined by

$$
M(P ; \lambda, \alpha)=\sup _{0<\rho<\frac{r}{2}} \lambda(B(P, \rho)) V(\rho) W(\rho) \rho^{\alpha-2} .
$$


The set

$$
\left\{P=(r, \Theta) \in \mathbf{R}^{n}-\{O\} ; M(P ; \lambda, \alpha) V^{-1}(r) W^{-1}(r) r^{2-\alpha}>\epsilon\right\}
$$

is denoted by $E(\epsilon ; \lambda, \alpha)$.

The following Theorems A and B give a way to estimate the Green-Sch potential and the Poisson-Sch integrals with measures on $C_{n}(\Omega)$ and $S_{n}(\Omega)$, respectively.

Theorem A Let $v$ be a positive measure on $C_{n}(\Omega)$ such that $G_{\Omega}^{a} v(P) \not \equiv+\infty(P=(r, \Omega)$ $\left.C_{n}(\Omega)\right)$ holds. Then for a sufficiently large $L$ we have

$$
\left\{P \in C_{n}(\Omega ;(L,+\infty)) ; G_{\Omega}^{a} \nu(P) \geq V(r)\right\} \subset E\left(\epsilon ; \mu^{\prime}, 1\right) .
$$

Theorem B Let $\mu$ be a positive measure on $S_{n}(\Omega)$ such that $P I_{\Omega}^{a} \mu(1) \not \equiv \vee(P=(r, \Theta) \in$ $C_{n}(\Omega)$ ). Then for a sufficiently large $L$ we have

$$
\left\{P \in C_{n}(\Omega ;(L,+\infty)) ; P I_{\Omega}^{a} \mu(P) \geq V(r)\right\} \subset E\left(\epsilon ; \mu^{\prime}, 1\right)
$$

It is known that the Martin boundary of $C_{n}(\Omega)$ is the se $\partial C_{n}(\Omega) \cup\{\infty\}$, each of which is a minimal Martin boundary point. For $\left.P \Omega C_{m}\right)$ and $\left(\chi \in \partial C_{n}(\Omega) \cup\{\infty\}\right.$, the Martin kernel can be defined by $M_{\Omega}^{a}(P, Q)$. If the reI nce p nt $P$ is chosen suitably, then we have

$$
\left.M_{\Omega}^{a}(P, \infty)=V(r) \varphi(\Theta) \text { and } N, r, \Omega\right)=c W(r) \varphi(\Theta)
$$

for any $P=(r, \Theta) \in C_{n}(\Omega)$.

In $[7,10]$, Xue and 7 nao-Yamada Introduce the notations of $a$-thin (with respect to the Schrödinger oper or $S c h_{a}$ ) at a point and $a$-rarefied sets at infinity (with respect to the Schrödinger ope $\quad s c h_{a}$ ), which generalized the earlier notations obtained by Miyamoto, Hosh u. olot (see [11-14]).

Defin; on (see 1/A) A set $H$ in $\mathbf{R}^{n}$ is said to be $a$-thin at a point $Q$ if there is a fine ne; s.bol $\mathrm{d} t \epsilon$ of $Q$ which does not intersect $H \backslash\{Q\}$. Otherwise $H$ is said to be not $a$-thin a. on conts.

Definition 2 (see [10]) A subset $H$ of $C_{n}(\Omega)$ is said to be $a$-rarefied at infinity on cones, if th re exists a positive superfunction $v(P)$ on cones such that

$$
\inf _{P \in C_{n}(\Omega)} \frac{v(P)}{M_{\Omega}^{a}(P, \infty)} \equiv 0
$$

and

$$
H \subset\left\{P=(r, \Theta) \in C_{n}(\Omega) ; v(P) \geq V(r)\right\}
$$

Let $H$ be a bounded subset of $C_{n}(\Omega)$. Then $\hat{R}_{M_{\Omega}^{a}(, \infty)}^{H}$ is bounded on cones and the greatest generalized harmonic minorant of $\hat{R}_{M_{\Omega}^{a}(\cdot, \infty)}^{H}$ is zero. We see from the Riesz decomposition 
theorem (see [6], Theorem 2) that there exists a unique positive measure $\lambda_{H}^{a}$ on cones such that (see [7], p.6)

$$
\hat{R}_{M_{\Omega}^{a}(\cdot, \infty)}^{H}(P)=G_{\Omega}^{a} \lambda_{H}^{a}(P)
$$

for any $P \in C_{n}(\Omega)$ and $\lambda_{H}^{a}$ is concentrated on $I_{H}$, where

$$
I_{H}=\left\{P \in C_{n}(\Omega) ; H \text { is not } a \text {-thin at } P\right\} .
$$

We denote the total mass $\lambda_{H}^{a}\left(C_{n}(\Omega)\right)$ of $\lambda_{H}^{a}$ by $\lambda_{\Omega}^{a}(H)$.

Recently, GX Xue (see [7], Theorem 2.5) gave a criterion for a subset $H$ of $C_{n}(2)$ to be a-rarefied set at infinity.

Theorem C $A$ subset $H$ of $C_{n}(\Omega)$ is a-rarefied at infinity on cones if an only if

$$
\sum_{j=0}^{\infty} W\left(2^{j}\right) \lambda_{H_{j}}^{a}\left(C_{n}(\Omega)\right)<\infty,
$$

where $H_{j}=H \cap C_{n}\left(\Omega ;\left[2^{j}, 2^{j+1}\right)\right)$ and $j=0,1,2, \ldots$

Our aim in this paper is to characterize the geometrica' property of $a$-rarefied sets at infinity.

Theorem 1 If a subset $H$ of $C_{n}(\Omega)$ is r-rarefied. ifinity on cones, then $H$ has a covering $\left\{r_{j}, R_{j}\right\}(j=0,1,2, \ldots)$ satisfying

$$
\sum_{j=0}^{\infty}\left(\frac{r_{j}}{R_{j}}\right) \frac{V\left(R_{j}\right)}{V\left(r_{j}\right)} \frac{W\left(R_{j}\right)}{\left.W / r_{j}\right)}<\infty .
$$

Next, we immediatel ve thle following result from Theorem 1.

Corollary 1 Let $1\left(P, v\right.$, positive superfunction on cones. Then $v(P) V^{-1}(r)$ uniformly converges to $\left.c_{\infty}, a\right) \varphi(\Theta)$ as $r \rightarrow \infty$ outside a set which has a covering $\left\{r_{j}, R_{j}\right\}(j=0,1,2, \ldots)$ satisyl, 1 .........ere

$$
(v, a)=\inf _{P \in C_{n}(\Omega)} \frac{v(P)}{M_{\Omega}^{a}(P, \infty)} .
$$

inally, we prove the following result.

Theorem 2 If a subset $H$ of $C_{n}(\Omega)$ has a covering $\left\{r_{j}, R_{j}\right\}(j=0,1,2, \ldots)$ satisfying $(1.5)$, then it is possible that $H$ is not a-rarefied at infinity on cones.

\section{Main lemmas}

Lemma 1 Let $\lambda$ be any positive measure on $\mathbf{R}^{n}$ having finite total mass. Then $E(\epsilon ; \lambda, 1)$ has a covering $\left\{r_{j}, R_{j}\right\}(j=1,2, \ldots)$ satisfying

$$
\sum_{j=1}^{\infty}\left(\frac{r_{j}}{R_{j}}\right) \frac{V\left(R_{j}\right) W\left(R_{j}\right)}{V\left(r_{j}\right) W\left(r_{j}\right)}<\infty .
$$




\section{Proof Set}

$$
E_{j}(\epsilon ; \lambda, 1)=\left\{P=(r, \Theta) \in E(\epsilon ; \lambda, 1): 2^{j} \leq r<2^{j+1}\right\} \quad(j=2,3,4, \ldots) .
$$

If $P=(r, \Theta) \in E_{j}(\epsilon ; \lambda, 1)$, then there exists a positive number $\rho(P)$ such that

$$
\left(\frac{\rho(P)}{r}\right) \frac{V(r) W(R)}{V(\rho(P)) W(\rho(P))} \approx\left(\frac{\rho(P)}{r}\right)^{n-1} \leq \frac{\lambda(B(P, \rho(P)))}{\epsilon} .
$$

Since $E_{j}(\epsilon ; \lambda, 1)$ can be covered by the union of a family of balls $\left\{B\left(P_{j, i}, \rho_{j, i}\right): \rho_{;} \in\right.$ $\left.E_{k}(\epsilon ; \lambda, 1)\right\}\left(\rho_{j, i}=\rho\left(P_{j, i}\right)\right)$. By the Vitali lemma (see [15]), there exists $\Lambda_{j} \subset E(\epsilon ; \lambda, 1)$, which is at most countable, such that $\left\{B\left(P_{j, i}, \rho_{j, i}\right): P_{j, i} \in \Lambda_{j}\right\}$ are disjoint and $\left.E_{j}(\lambda, 1) \subset\right)$ $\bigcup_{P_{j, i} \in \Lambda_{j}} B\left(P_{j, i}, 5 \rho_{j, i}\right)$. So

$$
\bigcup_{j=2}^{\infty} E_{j}(\epsilon ; \lambda, 1) \subset \bigcup_{j=2}^{\infty} \bigcup_{P_{j, i} \in \Lambda_{j}} B\left(P_{j, i}, 5 \rho_{j, i}\right) .
$$

On the other hand, note that

$$
\bigcup_{P_{j, i} \in \Lambda_{j}} B\left(P_{j, i}, \rho_{j, i}\right) \subset\left\{P=(r, \Theta): 2^{j-1} \leq r<2^{j+2}\right\}
$$

so that

$$
\begin{aligned}
\sum_{P_{j, i} \in \Lambda_{j}}\left(\frac{5 \rho_{j, i}}{\left|P_{j, i}\right|}\right) \frac{V\left(\left|P_{j, i}\right|\right) W\left(\left|P_{j, i}\right|\right)}{V\left(\rho_{j, i}\right) W(\rho ; j} & \approx\left(\frac{5 o_{j, i}}{P_{j, i} \mid}\right)^{n-1} \\
& -\rho^{n-1} \sum_{P_{j, i} \in \Lambda_{j}} \frac{\lambda\left(B\left(P_{j, i}, \rho_{j, i}\right)\right)}{\epsilon} \\
\leq & \frac{5^{n-1}}{\epsilon} \lambda\left(C_{n}\left(\Omega ;\left[2^{j-1}, 2^{j+2}\right)\right)\right) .
\end{aligned}
$$

Hence m btain

$$
\begin{aligned}
\sum_{i=1}^{\infty} \underset{P_{j, i} \in \Lambda_{j}}{\Delta}\left(\frac{\rho_{j, i}}{\left|P_{j, i}\right|}\right) \frac{V\left(\left|P_{j, i}\right|\right) W\left(\left|P_{j, i}\right|\right)}{V\left(\rho_{j, i}\right) W\left(\rho_{j, i}\right)} & \approx \sum_{j=1}^{\infty} \sum_{P_{j, i} \in \Lambda_{j}}\left(\frac{\rho_{j, i}}{\left|P_{j, i}\right|}\right)^{n-1} \\
& \leq \sum_{j=1}^{\infty} \frac{\lambda\left(C_{n}\left(\Omega ;\left[2^{j-1}, 2^{j+2}\right)\right)\right)}{\epsilon} \\
& \leq \frac{3 \lambda\left(\mathbf{R}^{n}\right)}{\epsilon}
\end{aligned}
$$

Since $E(\epsilon ; \lambda, 1) \cap\left\{P=(r, \Theta) \in \mathbf{R}^{n} ; r \geq 4\right\}=\bigcup_{j=2}^{\infty} E_{j}(\epsilon ; \lambda, 1)$. Then $E(\epsilon ; \lambda, 1)$ is finally covered by a sequence of balls $\left\{B\left(P_{j, i}, \rho_{j, i}\right), B\left(P_{1}, 6\right)\right\}(j=2,3, \ldots ; i=1,2, \ldots)$ satisfying

$$
\sum_{j, i}\left(\frac{\rho_{j, i}}{\left|P_{j, i}\right|}\right) \frac{V\left(\left|P_{j, i}\right|\right) W\left(\left|P_{j, i}\right|\right)}{V\left(\rho_{j, i}\right) W\left(\rho_{j, i}\right)} \approx \sum_{j, i}\left(\frac{\rho_{j, i}}{\left|P_{j, i}\right|}\right)^{n-1} \leq \frac{3 \lambda\left(\mathbf{R}^{n}\right)}{\epsilon}+6^{n-\alpha}<+\infty,
$$

where $B\left(P_{1}, 6\right)\left(P_{1}=(1,0, \ldots, 0) \in \mathbf{R}^{n}\right)$ is the ball which covers $\left\{P=(r, \Theta) \in \mathbf{R}^{n} ; r<4\right\}$. 


\section{Proof of Theorem 1}

Since $H$ is $a$-rarefied at infinity on cones, by Definition 2 there exists a positive superfunction $v(P)$ on cones such that (1.3) and (1.4) hold.

For this $v(P)$ there exists a unique positive measure $\mu^{\prime \prime}$ on $S_{n}(\Omega)$ and a unique positive measure $v^{\prime \prime}$ on cones such that (see [2], Theorem 3)

$$
v(P)=c_{0}(v, a) M_{\Omega}^{a}(P, O)+G_{\Omega}^{a} \nu^{\prime \prime}(P)+P I_{\Omega}^{a} \mu^{\prime \prime}(P),
$$

where

$$
c_{0}(v, a)=\inf _{P \in C_{n}(\Omega)} \frac{v(P)}{M_{\Omega}^{a}(P, O)} .
$$

Let us denote

$$
\begin{aligned}
& H_{1}=\left\{P=(r, \Theta) \in C_{n}(\Omega) ; c_{0}(v, a) M_{\Omega}^{a}(P, O) \geq \frac{V(r)}{3}\right\}, \\
& H_{2}=\left\{P=(r, \Theta) \in C_{n}(\Omega) ; G_{\Omega}^{a} \nu^{\prime \prime}(P) \geq \frac{V(r)}{3}\right\}
\end{aligned}
$$

and

$$
H_{3}=\left\{P=(r, \Theta) \in C_{n}(\Omega) ; P I_{\Omega}^{a} \mu^{\prime \prime}(P) \geq V /,\right.
$$

respectively.

Then we see from (1.4) that

$$
H \subset H_{1} \cup H_{2} \cup H_{3} \text {. }
$$

For each $H_{i}(i=1,2,3$ we kno $v$ that it has a covering. It is evident from the boundedness of $H_{1}$ that $H_{1}$ hac a cover. $\left.{ }_{1}, R_{1}\right\}$ satisfying

$$
\frac{r_{1}}{R_{1}}<\rho \text {. }
$$

vinen apply Theorems A and B with the measures $\mu$ and $v$ defined by $\mu=3 \mu$ " and $v v^{\prime \prime}$, restectively, we can find two positive constants $L$ and $\epsilon$ such that

$$
H_{2} \cap C_{n}(\Omega ;(L,+\infty)) \subset E\left(\epsilon ; \mu^{\prime}, 1\right)
$$

and

$$
H_{3} \cap C_{n}(\Omega ;(L,+\infty)) \subset E\left(\epsilon ; v^{\prime}, 1\right)
$$

respectively.

By Lemma 1 , these sets $E\left(\epsilon ; \mu^{\prime}, 1\right)$ and $E\left(\epsilon ; v^{\prime}, 1\right)$ have coverings $\left\{r_{j}^{(2)}, R_{j}^{(2)}\right\}(j=1,2, \ldots)$ and $\left\{r_{j}^{(3)}, R_{j}^{(3)}\right\}(j=1,2, \ldots)$ satisfying

$$
\sum_{j=1}^{\infty}\left(\frac{r_{j}^{(2)}}{R_{j}^{(2)}}\right) \frac{V\left(R_{j}^{(2)}\right) W\left(R_{j}^{(2)}\right)}{V\left(r_{j}^{(2)}\right) W\left(r_{j}^{(2)}\right)}<+\infty
$$


and

$$
\sum_{j=1}^{\infty}\left(\frac{r_{j}^{(3)}}{R_{j}^{(3)}}\right) \frac{V\left(R_{j}^{(3)}\right) W\left(R_{j}^{(3)}\right)}{V\left(r_{j}^{(3)}\right) W\left(r_{j}^{(3)}\right)}<+\infty,
$$

respectively.

Then $H_{2}$ and $H_{3}$ also have coverings $\left\{r_{j}^{(2)}, R_{j}^{(2)}\right\}(j=1,2, \ldots)$ and $\left\{r_{j}^{(3)}, R_{j}^{(3)}\right\}(j=1,2, \ldots)$ satisfying (3.4) and (3.5), respectively.

Thus by rearranging coverings $\left\{r_{1}, R_{1}\right\},\left\{r_{j}^{(2)}, R_{j}^{(2)}\right\}(j=1,2, \ldots)$ and $\left\{r_{j}^{(3)}, R_{j}^{(3)}\right\}(j=1,2, \ldots$, we know that the set $H$ has a covering $\left\{r_{j}, R_{j}\right\}(j=0,1,2, \ldots)$ from (3.2) and satisfi $<\mathrm{s}(1.5)$ from (3.3), (3.4), and (3.5).

Thus we complete the proof of Theorem 1.

\section{Proof of Theorem 2}

Put

$$
r_{j}=3 \cdot 2^{j-1} \cdot j^{\frac{1}{2-n}} \quad \text { and } \quad R_{j}=3 \cdot 2^{j-1} \quad(j=1,2,3, \ldots) .
$$

A covering $\left\{r_{j}, R_{j}\right\}$ satisfies

$$
\left.\sum_{j=1}^{\infty}\left(\frac{r_{j}}{R_{j}}\right) \frac{V\left(R_{j}\right)}{V\left(r_{j}\right)} \frac{W\left(R_{j}\right)}{W\left(r_{j}\right)} \leq c \sum_{j=1}^{\infty}\left(\frac{r_{j}}{R_{j}}\right)^{n-1}-c \sum_{i=1}\right)^{n-1}<+\infty
$$

from (1.2).

Let $C_{n}\left(\Omega^{\prime}\right)$ be a subset of $C_{r}(2)$, i.e. $D$. Suppose that this covering is so located: there is an integer $j_{0}$ such th $\mathrm{t} b_{j} \quad C_{n}\left(\Omega^{\prime}\right)$ and $R_{j}>2 r_{j}$ for $j \geq j_{0}$.

Next we shall prove th at the set $H \bigcup_{j=j_{0}}^{\infty} B_{j}$ is not $a$-rarefied at infinity on $C_{n}(\Omega)$. Since $\varphi(\Theta) \geq c$ for any $\Theta \in$ ', we have $M_{\Omega}^{a}(P, \infty) \geq c V\left(R_{j}\right)$ for any $P \in \bar{B}_{j}$, where $j \geq j_{0}$. Hence we have

$$
\hat{R}_{M_{j}^{a}}^{B_{j}}(P) \geq \operatorname{VV}(\mathrm{K} ; j)
$$

for ny $1 B_{j}$, wnere $j \geq j_{0}$.

'ke a me sure $\delta$ on cones, $\operatorname{supp} \delta \subset \bar{B}_{j}, \delta\left(\bar{B}_{j}\right)=1$ such that

$$
\int_{C_{n}(\Omega)}|P-Q|^{2-n} d \delta(P)=\left\{\operatorname{Cap}\left(\bar{B}_{j}\right)\right\}^{-1}
$$

for any $Q \in \bar{B}_{j}$, where Cap denotes the Newton capacity. Since

$$
G_{\Omega}^{a}(P, Q) \leq|P-Q|^{2-n}
$$

for any $P \in C_{n}(\Omega)$ and $Q \in C_{n}(\Omega)$,

$$
\begin{aligned}
\left\{\operatorname{Cap}\left(\bar{B}_{j}\right)\right\}^{-1} \lambda_{B_{j}}^{a}\left(C_{n}(\Omega)\right) & =\int\left(\int|P-Q|^{2-n} d \delta(P)\right) d \lambda_{B_{j}}^{a}(Q) \\
& \geq \int\left(\int G_{\Omega}^{a}(P, Q) d \lambda_{B_{j}}^{a}(Q)\right) d \delta(P)
\end{aligned}
$$




$$
\begin{aligned}
& =\int \hat{R}_{M_{\Omega}^{a}(\cdot, \infty)}^{B_{j}} d \delta(P) \\
& \geq c V\left(R_{j}\right) \delta\left(\bar{B}_{j}\right)=c V\left(R_{j}\right)
\end{aligned}
$$

from (4.1) and (4.2). Hence we have (see [5], p.1517)

$$
\lambda_{B_{j}}^{a}\left(C_{n}(\Omega)\right) \geq c \operatorname{Cap}\left(\bar{B}_{j}\right) V\left(R_{j}\right) \geq c r_{j}^{n-2} V\left(R_{j}\right)
$$

If we observe $\lambda_{H_{j}}^{a}\left(C_{n}(\Omega)\right)=\lambda_{B_{j}}^{a}\left(C_{n}(\Omega)\right)$, then we have by (1.2)

$$
\sum_{j=j_{0}}^{\infty} W\left(2^{j}\right) \lambda_{H_{j}}^{a}\left(C_{n}(\Omega)\right) \geq c \sum_{j=j_{0}}^{\infty}\left(\frac{r_{j}}{R_{j}}\right)^{n-2}=c \sum_{j=j_{0}}^{\infty} \frac{1}{j}=+\infty,
$$

from which it follows by Theorem $C$ that $H$ is not $a$-rarefied at infir ty

\section{Competing interests}

The authors declare that they have no competing interests.

\section{Authors' contributions}

Both authors contributed equally to the writing of this paper. Both authors read z $1 \mathrm{a}_{\mathrm{a}}$ proved the final manuscript.

\section{Author details}

${ }^{1}$ Institute of Management Science and Engineering, Henan nivers aifeng, 475001, China. ${ }^{2}$ Mathematics Institute, Roskilde University, Roskilde, 4000, Denmark.

\section{Acknowledgements}

This work was completed while the second autho sing the Department of Mathematical Sciences at the Columbia University, and he is grateful for th -...nd h tality or the Department. This work was partially supported by NSF Grant DMS-0913205.

Received: 23 January 2015 Accept d: 28 Ma, 15, Published online: 16 June 2015

\section{References}

1. Levin, B, Kheyfits, A: Asym, tic behavi br of subfunctions of time-independent Schrödinger operator. In: Some Topics on Value Distributio, Diff, entiability in Complex and P-Adic Analysis, Chapter 11, pp. 323-397. Science Press, Beijing (200

2. Qiao, L, Ren, YD: In ey r. scentations for the solutions of infinite order of the stationary Schrödinger equation in a cone. Monatshefte 12 n. 1, s(4), 593-603 (2014)

3. Verzhb imon, Ma 'ya, VG: Asymptotic behavior of solutions of elliptic equations of the second order close to a boy "ary. I. in Mat. -n. 12, 874-899 (1971)

4. Aiao, $. .20, \ldots$. ndary limits for fractional Poisson $a$-extensions of $L^{P}$ boundary function in a cone. Pac. J. Math. 272(1), 2 (2) $36,(2014)$

Diao, L, Pan, JS: Integral representations of generalized harmonic functions. Taiwan. J. Math. 17(5), 1503-1521 (2013)

6. L, Pan, GS: Generalization of the Phragmén-Lindelöf theorems for subfunctions. Int. J. Math. 24(8), 1350062 (20

7. Xue, GX: A remark on the a-minimally thin sets associated with the Schrödinger operator. Bound. Value Probl. 2014, $133(2014)$

Simon, B: Schrödinger semigroups. Bull. Am. Math. Soc. 7, 447-526 (1982)

9. Gilbarg, D, Trudinger, NS: Elliptic Partial Differential Equations of Second Order. Springer, Berlin (1977)

10. Zhao, T, Yamada, AJ: Growth properties of Green-Sch potentials at infinity. Bound. Value Probl. 2014, 245 (2014)

11. Brelot, M: On Topologies and Boundaries in Potential Theory. Lecture Notes in Mathematics, vol. 175. Springer, Berlin (1971)

12. Miyamoto, I, Yoshida, $\mathrm{H}$ : On harmonic majorization of the Martin function at infinity in a cone. Czechoslov. Math. J. 55(130)(4), 1041-1054 (2005)

13. Miyamoto, I, Yoshida, H: On a-minimally thin sets at infinity in a cone. Hiroshima Math. J. 37(1), 61-80 (2007)

14. Yoshida, $\mathrm{H}$ : Harmonic majorant of a radial subharmonic function on a strip and their applications. Int. J. Pure Appl. Math. 30(2), 259-286 (2006)

15. Stein, EM: Singular Integrals and Differentiability Properties of Functions. Princeton University Press, Princeton (1970) 